Trauma Berufskrankh 2014 - 16 [Suppl 4]:349-353 DOI 10.1007/s10039-014-2085-3

Online publiziert: 12. September 2014

(c) Springer-Verlag Berlin Heidelberg 2014

M. Faschingbauer $\cdot$ S. Kessler $\cdot$ C. Jürgens

Berufsgenossenschaftliches Unfallkrankenhaus Hamburg

\title{
Periprothetische
}

\section{Frakturen bei einliegender Hüfttotalendoprothese}

Vancouver-A-Frakturen. Es handelt sich um reine Trochanterfrakturen. Sie können in der Regel konservativ behandelt werden. Nur weit dislozierte Trochanterfrakturen bedürfen einer osteosynthetischen Versorgung mit z. B. Draht-Cerclagen oder speziellen krallenartigen Platten, welche den Trochanter refixieren.

Vancouver-B-Frakturen. Die verschiedenen Subtypen unterscheiden sich in einem wesentlichen Punkt. Bei B1-Frakturen ist die Prothese stabil, bei den B2- und B3-Frakturen ist sie gelockert, wobei bei den B2-Frakturen die Knochensubstanz gut ist und bei den B3-Frakturen zusätzlich zur Prothesenlockerung eine schlechte Knochensubstanz vorliegt.

Vancouver-C-Frakturen. Sie liegen unterhalb der Prothesenspitze und sind fast wie Oberschenkelfrakturen $\mathrm{zu}$ behandeln.

Ein Vorwurf an die Vancouver-Klassifikation war, dass der zeitliche Verlauf der Fraktur nicht berücksichtigt wird, denn frühe prothetische Frakturen (bis zur 6. Woche) sind anders zu behandeln als die späten Frakturen (nach 6 Wochen postoperativ). Bei den frühen VancouverB-Frakturen liegt in der Regel ein lockeres Implantat vor.

\section{Epidemiologie}

Im Mayo-Klinikregister wird über intraoperative periprothetische Femurfrakturen mit einer Häufigkeit von $0,3 \%$ bei zementierten Endoprothesen und von 5,4\% bei zementfreien Prothesen berichtet. Postoperativ liegt die Frakturrate bei der
Abb. 1 Vancouver-Klassifikation. (Mit freundl. Genehmigung der Firma Aesculap, B. Braun Melsungen $A G)$

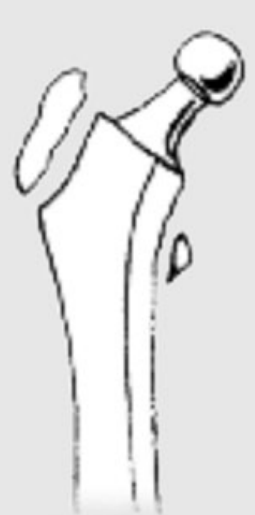

A

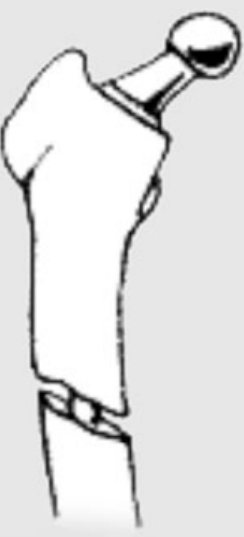

B1

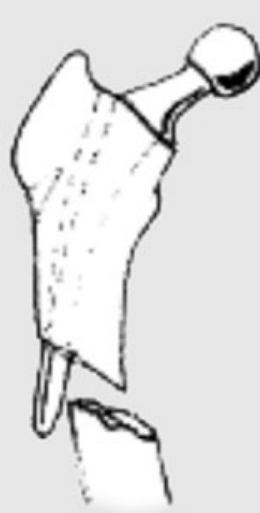

B2

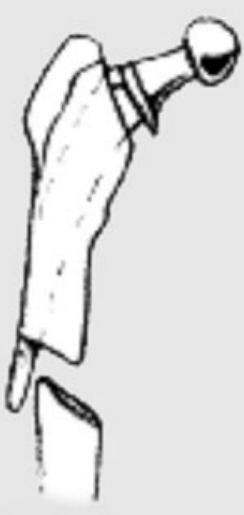

B3

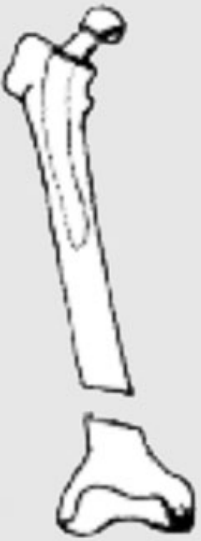

C 


\section{Periprothetische Frakturen}
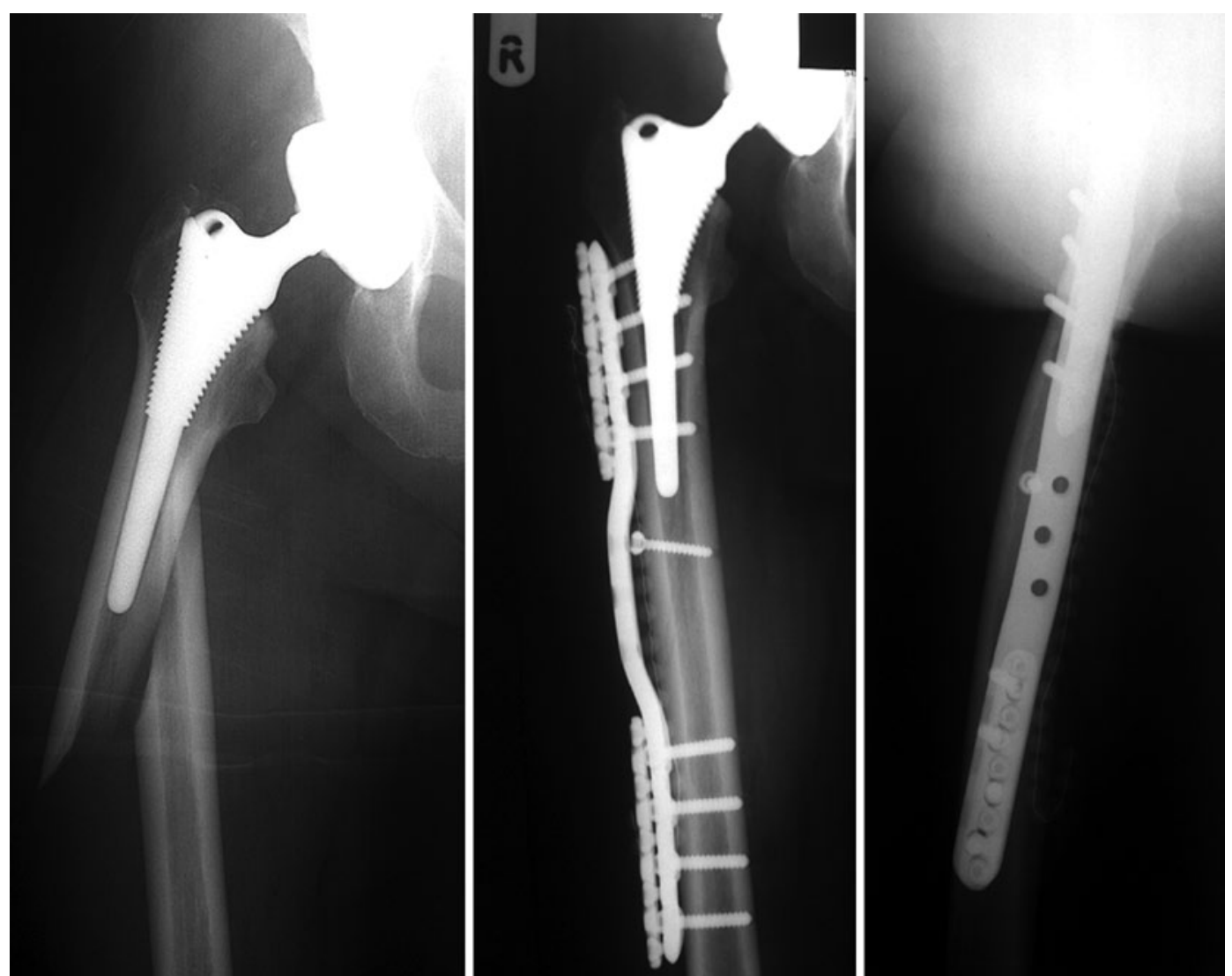

Abb. $2 \varangle$ Versorgung einer B1-Fraktur mit winkelstabilem Druckplattenfixateur
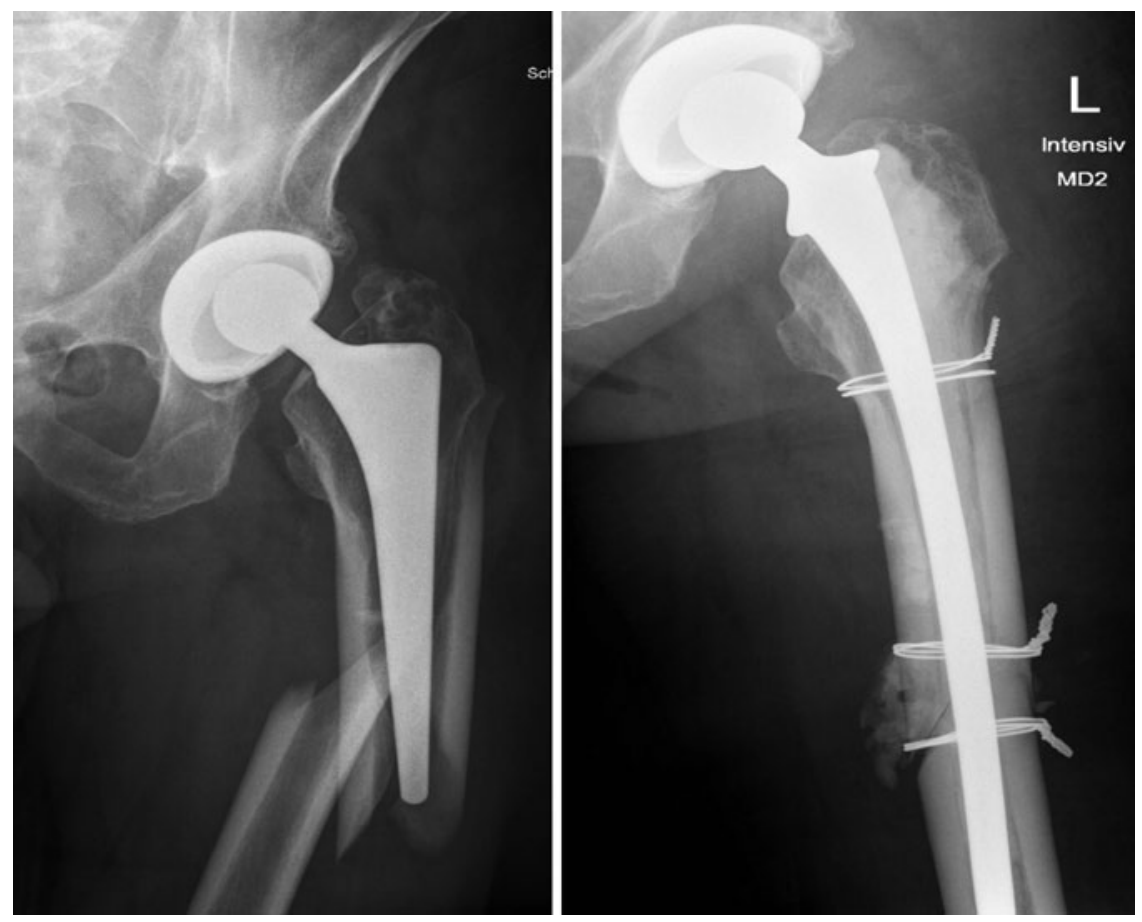

Abb. $3 \Delta$ B2-Fraktur mit gelockerter Prothese bei guter Knochenqualität
Primärendoprothetik bei etwa $1 \%$ und bei Revisionseingriffen bei 4\% [6].

Betrachtet man die Entwicklung der Häufigkeit im Laufe der Zeit, zeigen sich eine Zunahme der B2-Frakturen von 2003 zu 2012 um etwa das 3-Fache und eine gute Verdoppelung der B3- sowie der B1Frakuren. Die C- und A-Frakturen sind zahlenmäßig am geringsten.

Im Zeitraum von 1987-2003 betrug die Inzidenz periprothetischer Frakturen 0,14\%. Im Zeitraum von 2004-2007 stieg sie um das mehr als 5 -Fache auf $0,78 \%$ an [7]. Ob dieser Anstieg auf die frühe Mobilisation, das minimalinvasive Vorgehen mit kleinen Inzisionen, die verwendeten Implantate (proximal beschichtete Stems) oder den Operateur zurückzuführen ist, lässt sich nicht sicher sagen.

\section{Risiken}

Risikofaktoren für eine periprothetische Femurfraktur sind die verminderte Knochendichte bei der Osteoporose, die entzündliche Arthritis und andere Knochenerkrankungen. Eine Lockerung des Implantats wirkt sich ebenfalls risikoer- 
höhend aus, ebenso auch Revisionsoperationen mit Beeinträchtigung der Knochenqualität sowie Fehllagen der Implantate. Unter Letzteren sind die Varusfehlstellung oder ein inkompletter Zementmantel bei zementierter Prothese sowie die Entwicklung von Lysen durch Abrieb oder das Stress-Shielding zu nennen.

\section{Therapie}

Im Schwedischen Hüftendoprothetikregister haben Frakturen als Ursache für Revisionseingriffe einen Anteil von 7\%.

\section{Therapiewahl}

Kriterien für das therapeutische Vorgehen bei Patienten mit prothetischen Femurfrakturen sind die Lokalisation der Fraktur relativ zur Prothese, die Stabilität der Prothese und die Knochenqualität. Nicht zuletzt ist natürlich der Patient mit seinen Nebenerkrankungen und seiner Compliance von entscheidender Bedeutung. Ein Großteil dieser Kriterien wird durch die Vancouver-Klassifikation von Masri et al. [5] und Duncan u. Masri [1] abgedeckt.

\section{Typ-A-Frakturen}

Bei diesen Abrissen der Trochanteren ist in der Regel ein konservatives Vorgehen die Therapie der Wahl.

\section{Typ-B-Frakturen}

B1-Frakturen (- Abb. 2), also Frakturen im Prothesenbereich bei fest sitzender Prothese, sind die Domäne der Osteosynthese. Hier setzten sich winkelstabile Plattensysteme durch. Nichtwinkelstabile Systeme, wie die Oberschenkel-LCDC-Platte (LCDC: „limited contact dynamic compression"), erwiesen sich als nicht stabil genug [5]. Unter den winkelstabilen Systemen sind v. a. Platten mit der Möglichkeit eines polyaxialen Einbringens der Schrauben von Vorteil. Auch Platten mit zusätzlichen Cerclagen zeigen ein gewisses Revival, so wird die Ogden-Platte (Fa. Zimmer, Warsaw, IN, 1976), welche sich damals als nicht stabil genug erwies, erneut als Kombination von winkelstabilen Platten aufgelegt, mit der Möglichkeit, zusätzliche Cerclagen über die Platte einbringen zu können.
B2-Frakturen (• Abb. 3) sind durch eine Fraktur im Prothesenbereich mit gelockerter Prothese charakterisiert, wobei die Knochenqualität noch zufriedenstellend ist. Hier ist aufgrund der gelockerten Prothese deren Wechsel indiziert. Dieser ist sowohl zementfrei als auch zementiert möglich. Bei der zementfreien Version ist eine sichere Fixierung des Implantats, meist distal, notwendig.

Bei B3-Frakturen liegt zusätzlich zur gelockerten Prothese eine schlechte Knochenqualität vor. Hier verbleibt nur der Prothesenwechsel mit einer Revisionsoder Tumorprothese (• Abb. 4). Ob eine Augmentation mit Fremdknochen vorgenommen werden sollte, ist noch nicht sicher geklärt. Gerade in der amerikanischen Literatur wurde von hervorragenden Ergebnissen bei der Versorgung mit halbierten Fremdfemora, sog. Struts (- Abb. 5), berichtet. Diese werden teilweise nur mit Cerclagen um die Prothesen fixiert oder in Kombination mit einer Platte zur Stabilisierung benutzt. Haddad et al. [2] berichteten über hervorragende Ergebnisse mit dieser Methode. Es kommen "frozen“ oder "deep freeze femora“ zum Einsatz. In Deutschland sind nur sterilisierte, gefriergetrocknete Femora gesetzlich erlaubt. Ob diese ein anderes Einheilungsverhalten aufweisen, ist nicht bekannt.

Unsere Erfahrungen mit diesem Verfahren sind nicht besonders überzeugend. Im Endeffekt hat man das Gefühl, dass man riesige Sequester am Femur fixiert. Auch das Denudieren des Femurs (die Struts werden teilweise auch medial am Femur angelegt) entspricht nicht den Vorstellungen eines gewebeschonenden Vorgehens mit Erhalt der Weichteildurchblutung. Aber diesbezüglich ist sicherlich noch keine endgültige Aussage möglich, und weitere Ergebnisse müssen abgewartet werden.

Von 1999 bis Januar 2014 behandelten wir 88 Frakturen bei einliegenden Hüfttotalendoprothesen. Bei 31 Patienten handelte es sich um eine Vancouver-B1-Fraktur, die jeweils mit winkelstabilen Platten operativ versorgt wurde. Hierbei waren 6 Komplikationen zu verzeichnen: 2 Plattenausrisse, 1 Plattenbruch und 3 Frühinfektionen. Lindahl et al. [4] berichteten über eine Versagerquote von $33,9 \%$ bei
Trauma Berufskrankh 2014 • 16[Suppl 4]:349-353 DOI 10.1007/s10039-014-2085-3

(c) Springer-Verlag Berlin Heidelberg 2014

\section{Faschingbauer $\cdot$ S. Kessler $\cdot$ C. Jürgens Periprothetische Frakturen bei einliegender Hüfttotalendoprothese}

\section{Zusammenfassung}

Hintergrund. Periprothetische Frakturen nehmen an Häufigkeit zu.

Klassifikation. Eine therapieorientierte Einteilung für periprothetische Frakturen ist die Vancouver-Klassifikation.

Therapie. A-Frakturen werden in der Regel konservativ versorgt. C-Frakturen sind wie Schaftfrakturen zu behandeln. B1-Frakturen sind die Domäne der Plattenosteosynthese, B2- und B3-Frakturen bedürfen eines Prothesenwechsels, ggf. mit knöcherner Augmentation.

\section{Schlüsselwörter}

Periprothetische Fraktur .

Hüfttotalendoprothese - Vancouver-

Klassifikation · Plattenosteosynthese .

Prothesenwechsel

\section{Periprosthetic fractures in enclosed total hip arthroplasty}

\section{Abstract}

Background. Periprosthetic fractures are increasing in frequency.

Classification. A treatment-oriented classification is the Vancouver classification.

Therapy. Type A fractures will usually be treated conservatively, type $C$ fractures are treated in the same way as shaft fractures, type $B 1$ fractures are the domain of plate fixation and type $B 2$ and $B 3$ fractures require an exchange of the prosthesis possibly with bone augmentation.

\section{Keywords}

Periprosthetic fractures · Hip replacement arthroplasty · Vancouver classification - Plate osteosynthesis · Prosthesis exchange

mit Plattenosteosynthese und von 43,9\% bei nur mit Cerclagen versorgten periprothetischen Frakturen.

Zusammenfassend sind für die B1Frakturen Osteosynthesen mit winkelstabilen Plattensystemen eine gute Lösung, da gerade bei den polymorbiden Patienten eine kurze blutungsarme Operation gewählt werden kann. Insbesondere bei schlechter Knochenqualität zeigt die Winkelstabilität ihre Vorteile, v. a. wenn es 


\section{Periprothetische Frakturen}
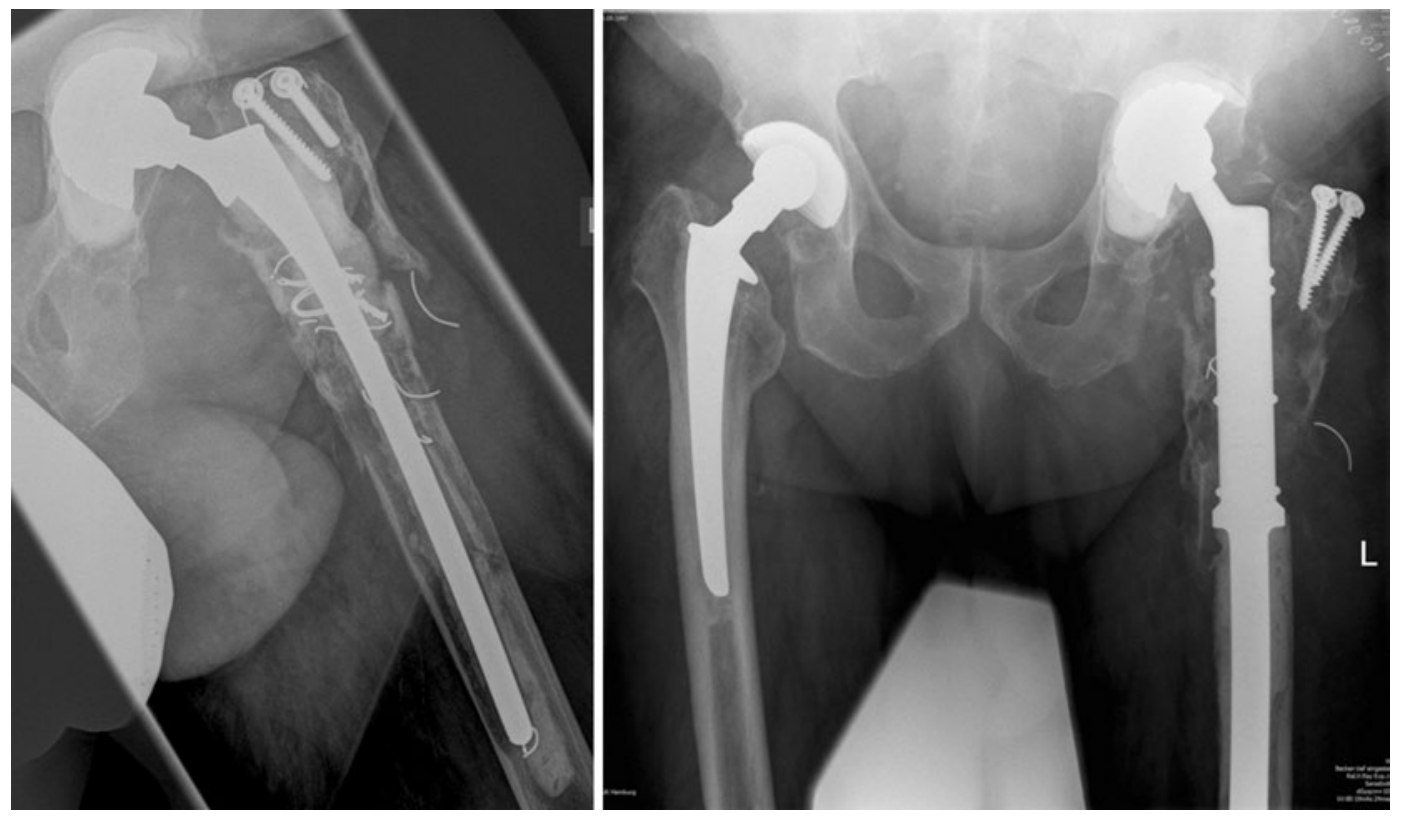

Abb. $4<$ Versorgung einer B3-Fraktur mit Prothesenlockerung und schlechter Knochenqualität mittels Tumorprothese

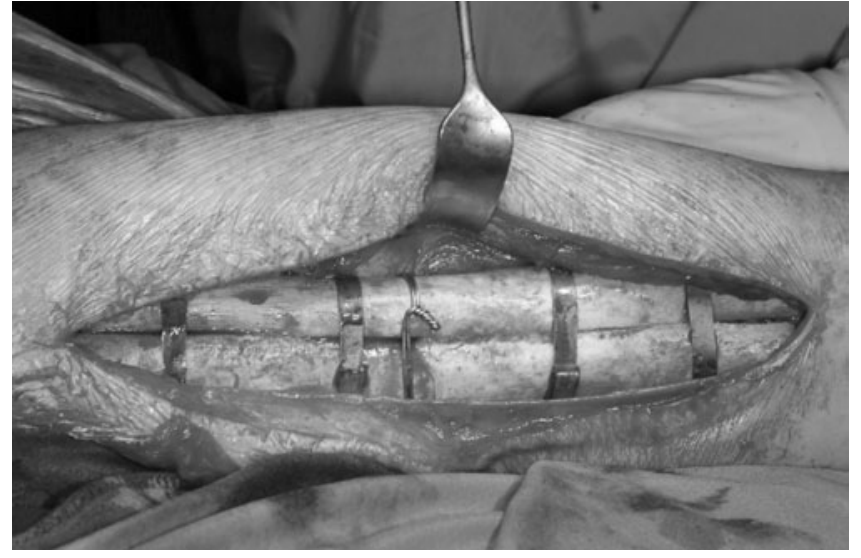

Abb. $5<$ Versorgung mit sog. Struts
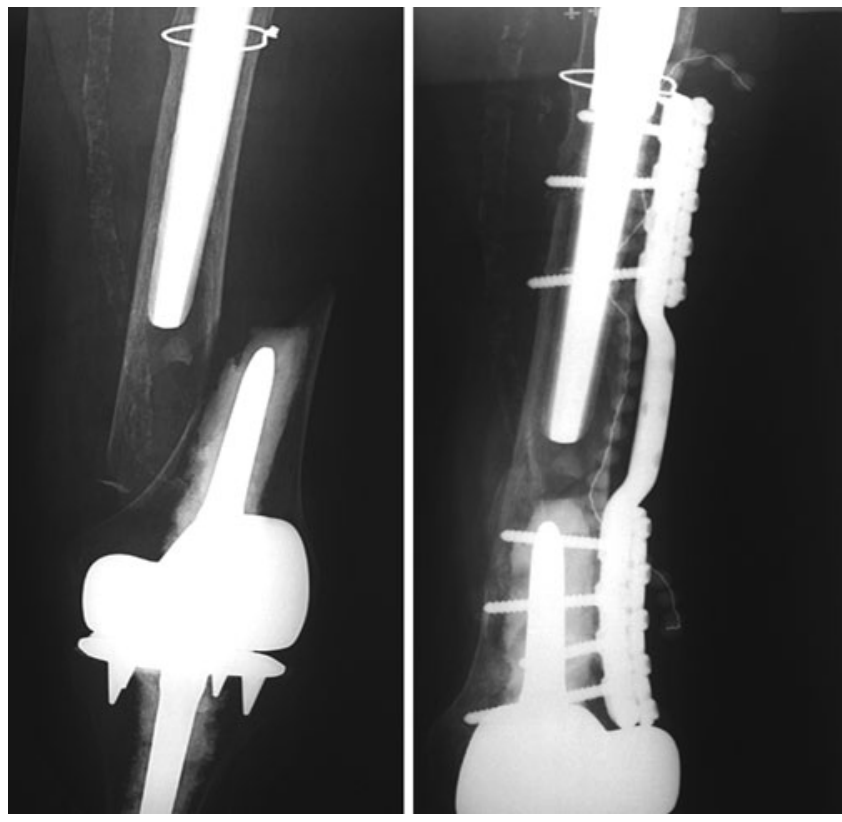

Abb. $6<$ Interprothetische Fraktur sich um Systeme handelt, die eine variable Positionierung der Schrauben und Platte erlauben. Somit können große Wechseloperationen vermieden werden.

Die Versorgung mit Fremdknochen im Sinne von Struts bei schlechter Knochenqualität bzw. bei Knochendefekten ist sicher überlegenswert. Autologen Knochen anzulegen, ist bei den alten Patienten häufig enttäuschend, da vom Beckenkamm kaum genügend brauchbare Spongiosa zu gewinnen ist. Ausreichende eigene Erfahrungen über Struts liegen aber noch nicht vor.

\section{Interprothetische Fraktur}

- Abb. 6 zeigt einen Sonderfall, eine sog. interprothetische Fraktur. Die Stressbelastung des Knochens ist insbesondere an der Stelle hoch, an der sich die Knie- und die Hüftendoprothese fast berühren, deshalb kommt es in diesem Bereich häufig zu Frakturen. Es besteht die Möglichkeit, mit einer winkelstabilen Platte eine stabile Osteosynthese zu erzielen, wobei natürlich zu überlegen ist, ob Knochen angelagert werden muss oder nicht. In diesem Fall wäre vielleicht doch die Versorgung mit einem Strut angebracht. In den USA wird dieser auch häufig medialseitig eingelegt, was aber mit einer ausgedehnten Devastierung des Knochens einhergeht. 


\section{Fazit für die Praxis}

- B1-Frakturen sind eine Domäne der Plattenosteosynthese. Hier ist die Winkelstabilität von Vorteil, kann aber sicher nicht jedes Problem lösen.

- B2-Frakturen erfordern einen Prothesenwechsel, wobei eine Spongiosaplastik in der Regel nicht nötig ist.

- Bei B3-Frakturen mit schlechter Knochenqualität verbleiben als Möglichkeiten entweder eine Revisionsprothese mit distaler Krafteinleitung oder eine Tumorprothese.

- Entschließt man sich zu einem Knochenersatz, wären Struts durchaus in Erwägung zu ziehen.

\section{Korrespondenzadresse}

\section{PD Dr. M. Faschingbauer}

Berufsgenossenschaftliches Unfallkrankenhaus Hamburg,

Bergedorfer Straße 10, 21033 Hamburg

m.faschingbauer@buk-hamburg.de

\section{Einhaltung ethischer Richtlinien}

Interessenkonflikt. M. Faschingbauer, S. Kessler und $\mathrm{Ch}$. Jürgens geben an, dass kein Interessenkonflikt besteht.

Dieser Beitrag beinhaltet keine Studien an Menschen oder Tieren.

The supplement containing this article is not sponsored by industry.

\section{Literatur}

1. Duncan CP, Masri BA (1995) Fractures of the femur after hip replacement. Instr Course Lect 44:293304

2. Haddad FS, Duncan CP, Berry DJ et al (2002) Cortical onlay allografts with or without a plate. J Bone Joint Surg Am 84:945-950

3. Lindahl $H$, Malchau $H$, Herberts $P$, Garellick $G$ (2005) Periprosthetic fractures classification and demographics of 1049 periprosthetic femoral fractures from the Swedish national hip arthroplasty register. J Arthroplasty 20:857-865

4. Lindahl H, Malchau H, Oden A, Garellick G (2006) Risk factors for failure after treatment of a periprosthetic fracture of the femur. J Bone Joint Surg $\mathrm{Br}$ 88:26-30

5. Masri BA, Meek RM, Duncan CP (2004) Periprosthetic fractures evaluation and treatment. Clin Orthop Relat Res 420:80-95

6. Pike J, Davidson D, Garbuz D et al (2009) Principles of treatment for periprosthetic femoral shaft fractures around well-fixed total hip arthroplasty. J Am Acad Orthop Surg 17:677-688
7. Ricci WM, Bolhofner BR, Loftus T et al (2006) Indirect reduction and plate fixation, without grafting, for periprosthetic femoral shaft fractures about a stable intramedullary implant. Surgical Technique. J Bone Joint Surg Am 88:275-282 